\title{
Enhancement and stabilization of traffic flow by moving in groups
}

\author{
Shingo Kurata and Takashi Nagatani* \\ Division of Thermal Science, Department of Mechanical Engineering, Shizuoka University, Hamamatsu 432-8561, Japan
}

(Received 20 November 2000; revised manuscript received 7 March 2001; published 13 June 2001)

\begin{abstract}
We study the traffic behavior of vehicles moving in groups analytically and numerically. A car-following model of traffic is extended to take into account a binary mixture of vehicles. It is shown that the movement in groups stabilizes the traffic flow. The jamming transition among the free traffic, the inhomogeneous traffic, and the homogeneous congested traffic occurs at a higher density than the threshold of the original model. The traffic current is highly enhanced at a high-density region by keeping a short headway without jam. The jamming transition is analyzed by using the linear stability method. It is found that the theoretical neutral stability curve agrees with the transition line obtained by the simulation.
\end{abstract}

DOI: 10.1103/PhysRevE.64.016106

PACS number(s): 05.70.Fh, 89.40.+k

\section{INTRODUCTION}

Recently, traffic problems have attracted considerable attention [1-3], due to the fact that traffic behavior is important in our life. Traffic flow is a kind of many-body system of strongly interacting vehicles. Traffic jams are typical signature of the complex behavior of traffic flow [4-10]. The jams obstruct traffic flow. In the result, the traffic current (flow) decreases significantly. For public demand, it will be necessary to enhance the traffic current and prevent the traffic jam. Traffic jams have been studied by several traffic models: car-following models, cellular automaton models, gas kinetic models, and hydrodynamic models [11-29].

We are interested in the enhancement of traffic flow and disappearance of traffic jam with the help of automatic control system. In the intelligent transportation system, several traffic systems are considered by communicating with other vehicles and controlling vehicles automatically with measuring instantly the headway. Recently, a concept of car in a virtual moving cell on an automated highway system is presented where vehicles move in groups and each group of vehicles constructs a platoon [30-32]. Both manual and automated vehicles are mixed on the highway. The automated vehicles move with keeping shorter headway than the manual vehicle. It is supposed that the traffic current enhances by keeping the short headway. However, the dynamical features of the system have been known little.

In this paper, we study the effect of the movement in groups on the traffic flow with the use of the car-following model. We would like to address whether or not the movement in groups enhances the traffic current and stabilizes the traffic flow. We investigate the jamming transition and the spatiotemporal structure of traffic jam. We calculate the fundamental diagram (the current-density relation) and the transition line numerically. We apply the linear stability method to the car-following model. We compare the neutral stability curve with the transition line.

\section{MODEL}

We present an extended version of the car-following models $[5,6,13]$. We describe the equations of motion for the

\footnotetext{
*Author to whom correspondence should be addressed.
}

system with vehicles moving in groups. We consider the traffic flow in which two kinds of vehicles exist on a highway: the one is the manual vehicle and the other is the automated vehicle. It is assumed that each kind of vehicle moves with optimal velocity different from the other. The automated vehicle keeps shorter headway than the manual vehicle. The manual vehicle has the higher velocity than the automated vehicle. The car-following model is described by the following equations of motion of manual vehicle $L$ and automated vehicle $T$ :

$$
\frac{d^{2} x_{n}}{d t^{2}}=a\left\{V_{n}\left(\Delta x_{n}\right)-\frac{d x_{n}}{d t}\right\},
$$

where $V_{n}\left(\Delta x_{n}\right)=V_{L}\left(\Delta x_{n}\right)$ or $V_{T}\left(\Delta x_{n}\right), V_{L}\left(\Delta x_{n}\right)$ is the optimal velocity for the manual car, $V_{T}\left(\Delta x_{n}\right)$ is the optimal velocity for the automated car, $x_{n}(t)$ is the position of vehicle $n$ at time $t, \Delta x_{n}(t)=x_{n+1}(t)-x_{n}(t)$ is the headway of vehicle $n$ at time $t$, and $a$ is the sensitivity (the inverse of the delay time).

A driver adjusts the car velocity to approach the optimal velocity determined by the observed headway. The sensitivity $a$ allows for the time lag $\tau=1 / a$ that it takes the car velocity to reach the optimal velocity when the traffic is varying. Generally, it is necessary that the optimal velocity function has the following properties: it is a monotonically increasing function and it has an upper bound (maximal velocity). We choose the maximal velocity and safety distance of manual vehicle to be twice of automated vehicle:

$$
\begin{gathered}
V_{L}(\Delta x)=\frac{2 \nu_{\max }}{2}\left[\tanh \left(\Delta x-2 x_{c}\right)+\tanh \left(2 x_{c}\right)\right], \\
V_{T}(\Delta x)=\frac{\nu_{\max }}{2}\left[\tanh \left(\Delta x-x_{c}\right)+\tanh \left(x_{c}\right)\right],
\end{gathered}
$$

where $v_{\max }$ is the maximal velocity of automated vehicle and $x_{c}$ is approximately the safety distance at the half of the maximal velocity of automated vehicle.

Fujioka, Omae, and Miyake [30] have presented the controllers for platooning (group) controls in the group movement. Their controllers keep the short headway for the automated vehicles and give the rapid response. They have 
A group of three vehicles

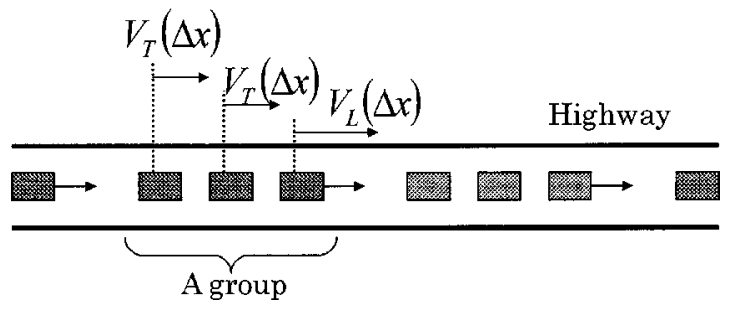

FIG. 1. Schematic illustration of our model for the group movement of three vehicles per group. All vehicles are partitioned into three vehicles in which the leading vehicle is the manual car and the second and third vehicles are the automated cars.

studied such a system that the automated vehicle differs from the manual vehicle by a shorter headway for congested traffic and by a high response.

The automated vehicle has a shorter reaction time than that of the manual vehicle. The reaction time corresponds to the inverse of the sensitivity in the optimal velocity model $[5,6,13]$. In Secs. III and IV, we will show that the difference of the sensitivities between the automated and manual vehicles does not have a significant effect on the traffic behavior. The traffic current depends little on the reaction time but mainly on the headway. We set $a_{T}=a_{L}$ and safety distance of manual vehicle to be twice of automated vehicle where $a_{T}$ and $a_{L}$ are the sensitivities of the automated and manual vehicles, respectively.

The reaction time of vehicle is given by the sum of the human and vehicle's response times. The vehicle's response time is determined by the vehicle's inertia. In the automated vehicle, one is able to reduce the human response time but the vehicle's response time is the same as the manual vehicle if their masses are the same. In order to shorten the safety distance of the automated vehicle, it is necessary to lower its maximal velocity because the automated vehicle avoids a collision. We choose the maximal velocity of the automated vehicle to be half of manual vehicle. If the maximal velocity of automated vehicle increases, the current enhancement becomes better than the case considered here.

For an example, we consider the movement in groups consisting of three vehicles. A group includes a leading manual vehicle and two tracking automated vehicles. Figure 1 shows the schematic illustration of model for the group movement of three vehicles. For simplicity, we assume that all vehicles are partitioned into three vehicles in which the leading vehicle is the manual car and the second and third vehicles are the automated cars. The equations of motion for three vehicles per group are given by

$$
\begin{gathered}
\frac{d^{2} x_{3 m}}{d t^{2}}=a\left[V_{L}\left(\Delta x_{3 m}\right)-\frac{d x_{3 m}}{d t}\right], \\
\frac{d^{2} x_{3 m-1}}{d t^{2}}=a\left[V_{T}\left(\Delta x_{3 m-1}\right)-\frac{d x_{3 m-1}}{d t}\right], \\
\frac{d^{2} x_{3 m-2}}{d t^{2}}=a\left[V_{T}\left(\Delta x_{3 m-2}\right)-\frac{d x_{3 m-2}}{d t}\right],
\end{gathered}
$$

where $m$ is the index indicating the group number.
Generally, the equations of motion for $n$ vehicles per group are described by

$$
\begin{gathered}
\frac{d^{2} x_{n m}}{d t^{2}}=a\left[V_{L}\left(\Delta x_{n m}\right)-\frac{d x_{n m}}{d t}\right] \\
\frac{d^{2} x_{n m-1}}{d t^{2}}=a\left[V_{T}\left(\Delta x_{n m-1}\right)-\frac{d x_{n m-1}}{d t}\right], \\
\frac{d^{2} x_{n m-2}}{d t^{2}}=a\left[V_{T}\left(\Delta x_{n m-2}\right)-\frac{d x_{n m-2}}{d t}\right] \\
\frac{d^{2} x_{n m-(n-1)}}{d t^{2}}=a\left[V_{T}\left(\Delta x_{n m-(n-1)}\right)-\frac{d x_{n m-(n-1)}}{d t}\right] .
\end{gathered}
$$

Equations (4) describe the group movement with the periodic configuration.

\section{LINEAR STABILITY ANALYSIS}

We apply the linear stability analysis to the model described by Eq. (4). We consider the stability of the traffic flow in which all vehicles move with the same velocity. The traffic flow is a solution at the steady state for Eq. (4). First, we consider the group movement of two vehicles per group where the manual vehicles are positioned alternately to the automated vehicles. The equations of motion are given by

$$
\begin{gathered}
\frac{d^{2} x_{2 m}}{d t^{2}}=a\left[V_{L}\left(\Delta x_{2 m}\right)-\frac{d x_{2 m}}{d t}\right], \\
\frac{d^{2} x_{2 m-1}}{d t^{2}}=a\left[V_{T}\left(\Delta x_{2 m-1}\right)-\frac{d x_{2 m-1}}{d t}\right] .
\end{gathered}
$$

Solutions of uniform-velocity traffic are given by

$$
\begin{gathered}
x_{2 m}^{(0)}=V_{L}\left(\Delta x_{L}^{(0)}\right) t+(m-1) \Delta x_{L}^{(0)}+m \Delta x_{T}^{(0)}, \\
x_{2 m-1}^{(0)}=V_{T}\left(\Delta x_{T}^{(0)}\right) t+(m-1)\left(\Delta x_{L}^{(0)}+\Delta x_{T}^{(0)}\right),
\end{gathered}
$$

where $V_{L}\left(\Delta x_{L}^{(0)}\right)=V_{T}\left(\Delta x_{T}^{(0)}\right)$, the average headway $\Delta x^{(0)}$ is given by $\Delta x^{(0)}=\left(\Delta x_{L}^{(0)}+\Delta x_{T}^{(0)}\right) / 2$.

Let us add small disturbances to the steady-state solution (6). We study whether or not the disturbances are amplified with time. One takes the solutions of Eq. (5) as follows:

$$
\begin{gathered}
x_{2 m}=x_{2 m}^{(0)}+y_{2 m}, \quad\left|y_{2 m}\right| \ll 1, \\
x_{2 m-1}=x_{2 m-1}^{(0)}+y_{2 m-1}, \quad\left|y_{2 m-1}\right| \ll 1 .
\end{gathered}
$$

Then, the linear equations are obtained 


$$
\begin{gathered}
\frac{d^{2} y_{2 m}}{d t^{2}}=a\left[\Delta y_{2 m} V_{L}^{\prime}\left(\Delta x_{L}^{(0)}\right)-\frac{d y_{2 m}}{d t}\right], \\
\frac{d^{2} y_{2 m-1}}{d t^{2}}=a\left[\Delta y_{2 m-1} V_{T}^{\prime}\left(\Delta x_{T}^{(0)}\right)-\frac{d y_{2 m-1}}{d t}\right],
\end{gathered}
$$

where

$$
\begin{gathered}
V_{L}^{\prime}\left(\Delta x_{L}^{(0)}\right)=\left.\frac{d V_{L}\left(\Delta x_{n}\right)}{d \Delta x_{n}}\right|_{\Delta x_{n}=\Delta x_{L}^{(0)}} \text { and } \\
V_{T}^{\prime}\left(\Delta x_{T}^{(0)}\right)=\left.\frac{d V_{T}\left(\Delta x_{n}\right)}{d \Delta x_{n}}\right|_{\Delta x_{n}=\Delta x_{\tau}^{(0)}} .
\end{gathered}
$$

By expanding

$$
\begin{gathered}
y_{2 m}(t)=Y_{L} \exp \{i k(2 m)+\omega t\}, \\
y_{2 m-1}(t)=Y_{T} \exp \{i k(2 m-1)+\omega t\}
\end{gathered}
$$

and replacing those into Eq. (8), one obtains

$$
\left[\begin{array}{ll}
M_{1} & M_{2} \\
M_{3} & M_{4}
\end{array}\right]\left\{\begin{array}{l}
Y_{L} \\
Y_{T}
\end{array}\right\}=\left\{\begin{array}{l}
0 \\
0
\end{array}\right\}
$$

with

$$
\begin{gathered}
M_{1}=\omega^{2}+a \omega+a V_{L}^{\prime}\left(\Delta x_{L}^{(0)}\right), \\
M_{2}=-a V_{L}^{\prime}\left(\Delta x_{L}^{(0)}\right) e^{i k}, \\
M_{3}=-a V_{T}^{\prime}\left(\Delta x_{T}^{(0)}\right) e^{i k}, \\
M_{4}=\omega^{2}+a \omega+a V_{T}^{\prime}\left(\Delta x_{T}^{(0)}\right) .
\end{gathered}
$$

For $Y_{L}$ and $Y_{T}$ to have nontrivial solutions, it should be satisfied that the determinant is zero. One obtains

$$
\begin{aligned}
\omega^{4}+ & 2 a \omega^{3}+a\left\{a+V_{L}^{\prime}\left(\Delta x_{L}^{(0)}\right)+V_{T}^{\prime}\left(\Delta x_{T}^{(0)}\right)\right\} \omega^{2} \\
& +a^{2}\left\{V_{L}^{\prime}\left(\Delta x_{L}^{(0)}\right)+V_{T}^{\prime}\left(\Delta x_{T}^{(0)}\right)\right\} \omega \\
& -a^{2}\left(e^{2 i k}-1\right) V_{L}^{\prime}\left(\Delta x_{L}^{(0)}\right) V_{T}^{\prime}\left(\Delta x_{T}^{(0)}\right)=0 .
\end{aligned}
$$

By solving Eq. (10) for $\omega$, one finds that the leading term of $\omega$ is on the order of $i k$. If $i k$ approaches zero, $\omega \rightarrow 0$. Let us derive the long-wave expansion of $\omega$, which is determined order by order around $i k \approx 0$. By expanding $\omega=\omega_{1} i k$ $+\omega_{2}(i k)^{2}+\cdots$, one obtains

$$
\begin{gathered}
\omega_{1}=\frac{2 V_{L}^{\prime}\left(\Delta x_{L}^{(0)}\right) V_{T}^{\prime}\left(\Delta x_{T}^{(0)}\right)}{V_{L}^{\prime}\left(\Delta x_{L}^{(0)}\right)+V_{T}^{\prime}\left(\Delta x_{T}^{(0)}\right)} \\
\omega_{2}=\frac{2 V_{L}^{\prime}\left(\Delta x_{L}^{(0)}\right) V_{T}^{\prime}\left(\Delta x_{T}^{(0)}\right)}{\left\{V_{L}^{\prime}\left(\Delta x_{L}^{(0)}\right)+V_{T}^{\prime}\left(\Delta x_{T}^{(0)}\right)\right\}^{3}}\left[a ^ { 2 } \left\{V_{L}^{\prime 2}\left(\Delta x_{L}^{(0)}\right)\right.\right. \\
\left.+V_{T}^{\prime 2}\left(\Delta x_{T}^{(0)}\right)\right\}-2 a V_{L}^{\prime}\left(\Delta x_{L}^{(0)}\right) V_{T}^{\prime}\left(\Delta x_{T}^{(0)}\right)\left\{V_{L}^{\prime}\left(\Delta x_{L}^{(0)}\right)\right. \\
\left.\left.+V_{T}^{\prime}\left(\Delta x_{T}^{(0)}\right)\right\}\right] .
\end{gathered}
$$

If $\omega_{2}$ is a negative value, the steady-state flow (6) becomes unstable for long-wavelength modes. When $\omega_{2}$ is a positive value, the steady-state flow is stable. The neutral stability condition is given by

$$
a=\frac{2 V_{L}^{\prime}\left(\Delta x_{L}^{(0)}\right) V_{T}^{\prime}\left(\Delta x_{T}^{(0)}\right)\left\{V_{L}^{\prime}\left(\Delta x_{L}^{(0)}\right)+V_{T}^{\prime}\left(\Delta x_{T}^{(0)}\right)\right\}}{V_{L}^{\prime 2}\left(\Delta x_{L}^{(0)}\right)+V_{T}^{\prime 2}\left(\Delta x_{T}^{(0)}\right)} .
$$

We consider the group movement of three vehicles per group shown in Fig. 1. The set of motion equations is given by Eq. (3). Their equations have the following solutions of the uniform-velocity flow:

$$
\begin{gathered}
x_{3 m}^{(0)}=V_{L}\left(\Delta x_{L}^{(0)}\right) t+(m-1) \Delta x_{L}^{(0)}+2 m \Delta x_{T}^{(0)}, \\
x_{3 m-1}^{(0)}=V_{T}\left(\Delta x_{T}^{(0)}\right) t+(m-1) \Delta x_{L}^{(0)}+(2 m-1) \Delta x_{T}^{(0)}, \\
x_{3 m-2}^{(0)}=V_{T}\left(\Delta x_{T}^{(0)}\right) t+(m-1)\left(\Delta x_{L}^{(0)}+2 \Delta x_{T}^{(0)}\right),
\end{gathered}
$$

where $V_{L}\left(\Delta x_{L}^{(0)}\right)=V_{T}\left(\Delta x_{T}^{(0)}\right)$, the average headway $\Delta x^{(0)}$ is given by $\Delta x^{(0)}=\left(\Delta x_{L}^{(0)}+2 \Delta x_{T}^{(0)}\right) / 3$.

Let us add small disturbances to the steady-state solution (14). We study whether or not the disturbances are amplified with time. One takes the solutions of Eq. (3) as follow:

$$
\begin{gathered}
x_{3}=x_{3 m}^{(0)}+y_{3 m}, \quad\left|y_{3 m}\right| \ll 1, \\
x_{3 m-1}=x_{3 m-1}^{(0)}+y_{3 m-1}, \quad\left|y_{3 m-1}\right| \ll 1, \\
x_{3 m-3}=x_{3 m-2}^{(0)}+y_{3 m-2}, \quad\left|y_{3 m-2}\right| \ll 1 .
\end{gathered}
$$

Then, the linear equations are obtained

$$
\begin{gathered}
\frac{d^{2} y_{3 m}}{d t^{2}}=a\left[\Delta y_{3 m} V_{L}^{\prime}\left(\Delta x_{L}^{(0)}\right)-\frac{d y_{3 m}}{d t}\right], \\
\frac{d^{2} y_{3 m-1}}{d t^{2}}=a\left[\Delta y_{3 m-1} V_{T}^{\prime}\left(\Delta x_{T}^{(0)}\right)-\frac{d y_{3 m-1}}{d t}\right], \\
\frac{d^{2} y_{3 m-2}}{d t^{2}}=a\left[\Delta y_{3 m-2} V_{T}^{\prime}\left(\Delta x_{T}^{(0)}\right)-\frac{d y_{3 m-2}}{d t}\right],
\end{gathered}
$$

where

$$
\begin{gathered}
V_{L}^{\prime}\left(\Delta x_{L}^{(0)}\right)=\left.\frac{d V_{L}\left(\Delta x_{n}\right)}{d \Delta x_{n}}\right|_{\Delta x_{n}=\Delta x_{L}^{(0)}} \text { and } \\
V_{T}^{\prime}\left(\Delta x_{T}^{(0)}\right)=\left.\frac{d V_{T}\left(\Delta x_{n}\right)}{d \Delta d x_{n}}\right|_{\Delta x_{n}=\Delta x_{T}^{(0)}} .
\end{gathered}
$$

By expanding 


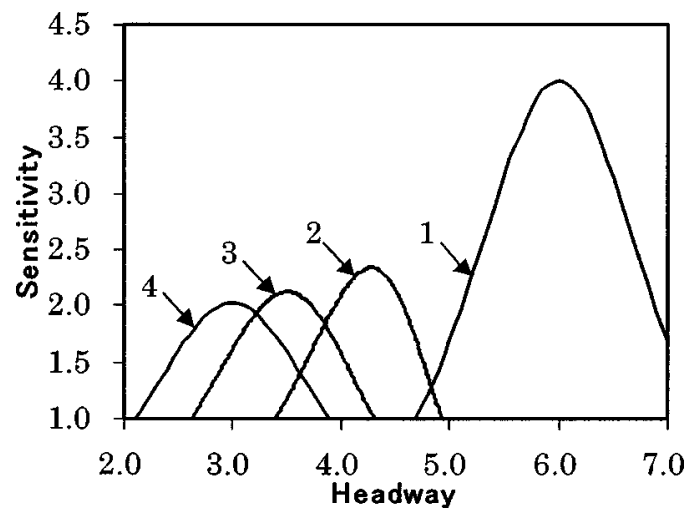

FIG. 2. Neutral stability curves in the headway-sensitivity space for (1) all manual vehicles, (2) the group movement of two vehicles per group, (3) the group movement of five vehicles per group, and (4) all automated vehicles, where $v_{\max }=2.0$ and $x_{c}=3.0$.

$$
\begin{gathered}
y_{3 m}(t)=Y_{L} \exp \{i k(3 m)+\omega t\}, \\
y_{3 m-1}(t)=Y_{T_{1}} \exp \{i k(3 m-1)+\omega t\}, \\
y_{3 m-2}(t)=Y_{T_{2}} \exp \{i k(3 m-2)+\omega t\}
\end{gathered}
$$

and replacing those into Eq. (16), one obtains

$$
\left[\begin{array}{ccc}
M_{1} & 0 & M_{2} \\
M_{4} & M_{3} & 0 \\
0 & M_{4} & M_{3}
\end{array}\right]\left\{\begin{array}{c}
Y_{L} \\
Y_{T_{1}} \\
Y_{T_{2}}
\end{array}\right\}=\left\{\begin{array}{l}
0 \\
0 \\
0
\end{array}\right\},
$$

with

$$
\begin{gathered}
M_{1}=\omega^{2}+a \omega+a V_{L}^{\prime}\left(\Delta x_{L}^{(0)}\right), \\
M_{2}=-a V_{L}^{\prime}\left(\Delta x_{L}^{(0)}\right) e^{i k}, \\
M_{3}=\omega^{2}+a \omega+a V_{T}^{\prime}\left(\Delta x_{T}^{(0)}\right), \\
M_{4}=-a V_{T}^{\prime}\left(\Delta x_{T}^{(0)}\right) e^{i k} .
\end{gathered}
$$

Similarly to the case of two vehicles, we obtain the following neutral stability condition for three vehicles:

$$
a=\frac{2 V_{L}^{\prime}\left(\Delta x_{L}^{(0)}\right) V_{T}^{\prime}\left(\Delta x_{T}^{(0)}\right)\left\{2 V_{L}^{\prime}\left(\Delta x_{L}^{(0)}\right)+V_{T}^{\prime}\left(\Delta x_{T}^{(0)}\right)\right\}}{2 V_{L}^{\prime 2}\left(\Delta x_{L}^{(0)}\right)+V_{T}^{\prime 2}\left(\Delta x_{T}^{(0)}\right)} .
$$

We derive the stability condition for the group movement of $n$ vehicles per group. The set of equations of motion for $n$ vehicles is given by Eq. (4). Similarly, one obtains the neutral stability condition for the movement of $n$ vehicles:

$$
a=\frac{2 V_{L}^{\prime}\left(\Delta x_{L}^{(0)}\right) V_{T}^{\prime}\left(\Delta x_{T}^{(0)}\right)\left\{(n-1) V_{L}^{\prime}\left(\Delta x_{L}^{(0)}\right)+V_{T}^{\prime}\left(\Delta x_{T}^{(0)}\right)\right\}}{(n-1) V_{L}^{\prime 2}\left(\Delta x_{L}^{(0)}\right)+V_{T}^{\prime 2}\left(\Delta x_{T}^{(0)}\right)}
$$

We show the neutral stability lines in Fig. 2. The neutral stability curves are shown for two cases of two and five vehicles where $v_{\max }=2.0$ and $x_{c}=3.0$. For comparison, the neutral stability lines are also indicated for the two limiting cases of all manual and all automated vehicles. Above each neutral stability curve, the uniform-velocity flow is stable. For any initial condition, all vehicles approach the traffic state with the same velocity. The uniform flow becomes unstable below each curve. Each apex of neutral stability curves indicates the critical point. Above the critical point, the uniform flow is always stable for any density. With increasing vehicles per group, the unstable region shifts to high-density side and shrinks. Therefore, the group movement stabilizes the traffic flow. The increase of vehicles per group strengthens the stabilization effect.

We would like to estimate roughly the current enhancement effect by moving in groups. We study the traffic current of group movement when the uniform-velocity state maintains without traffic jams. The current of traffic flow with no jams gives approximately the flow quantity except for the coexisting phase with jams. It is easy to calculate the traffic current without jams. For the traffic flow without jams, all vehicles move with the same velocity. Then, the velocity is given by the optimal velocity. The headways of manual and automated vehicles should satisfy the relationships

$$
\begin{gathered}
V_{L}\left(\Delta x_{L}^{(0)}\right)=V_{T}\left(\Delta x_{T}^{(0)}\right) \quad \text { and } \\
\Delta x^{(0)}=\left[\Delta x_{L}^{(0)}+(n-1) \Delta x_{T}^{(0)}\right] / n,
\end{gathered}
$$

where the optimal velocity function $V$ is given by Eq. (2) and $\Delta x^{(0)}$ is the average headway.

We define the relationship between the headway and the density as following: $\Delta x^{(0)}=1 / \rho-1$. If one adopts $\Delta x^{(0)}$ $=1 / \rho$, the density is larger than 1 for $\Delta x^{(0)}<1$ and diverges in the limit $\Delta x^{(0)} \rightarrow 0$. We adopt the relationship $\Delta x^{(0)}$ $=1 / \rho-1$ in order to normalize the density. This relation corresponds to a vehicle length of 1 . In the traffic flow without jams, the current is given by

$$
Q=V_{L}\left(\Delta x_{L}^{(0)}\right) /\left(\Delta x^{(0)}+1\right)
$$

where the headway of manual vehicle $L$ is determined by Eq. (20).

We show the current (21) for all manual vehicles, two vehicles per group, five vehicles per group, and all automated vehicles in Fig. 3 where $v_{\max }=2.0$ and $x_{c}=3.0$. For two vehicles per group, the current at low-density region is less than that of all manual vehicles since the maximal velocity of manual vehicle is twice that of automated vehicle. However, at high-density region, the current of two vehicles is higher than that of all manual vehicles. With increasing vehicles per group, the current increases highly at highdensity region. We find that the group movement has a significant effect on the current enhancement at high-density region.

As can be seen from Fig. 3, even higher maximal flow is obtained with only manual vehicles. However, at a higher density than 0.1 , the current decreases rapidly and the traffic exhibits the low current. In order to enhance the current at the higher density, it is necessary to move with a shorter headway. Generally, to keep the short headway, the safety 


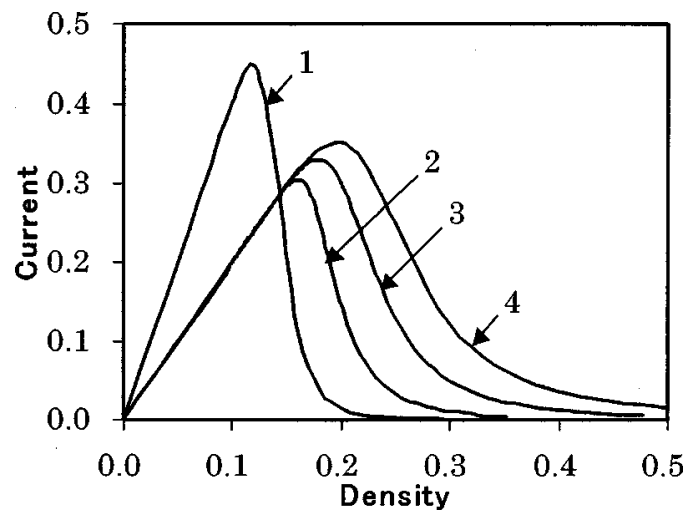

FIG. 3. Plots of the traffic current without jams against density for (1) all manual vehicles, (2) two vehicles per group, (3) five vehicles per group, and (4) all automated vehicles, where $v_{\max }$ $=2.0$ and $x_{c}=3.0$.

distance $x_{c}$ must be short. In order to shorten the safety distance, the maximal velocity should be low due to the vehicle inertia. By keeping the short headway, the traffic current can be enhanced at a high density. Also, the automated vehicle is driven with a higher sensitivity to keep the short headway. We study the effect of the sensitivity of automated vehicle on the traffic stability. For two vehicles per group, one obtains the neutral stability condition similarly to Eq. (13),

$$
a_{L}=\frac{2 V_{L}^{\prime}\left(\Delta x_{L}^{(0)}\right) V_{T}^{\prime}\left(\Delta x_{T}^{(0)}\right)\left\{V_{L}^{\prime}\left(\Delta x_{L}^{(0)}\right)+\frac{a_{r}}{a_{L}} V_{T}^{\prime}\left(\Delta x_{T}^{(0)}\right)\right\}}{\frac{a_{T}}{a_{L}}\left\{V_{L}^{\prime 2}\left(\Delta x_{L}^{(0)}\right)+V_{T}^{\prime 2}\left(\Delta x_{T}^{(0)}\right)\right\}},
$$

where $a_{L}$ and $a_{T}$ are the sensitivities of the manual and automated vehicles respectively. Figure 4 shows the neutral stability lines for $a_{T} / a_{L}=1.0,1.5$, and 2.0. With increasing the sensitivity $a_{T}$ of the automated vehicle, the neutral stability curve becomes a little low but the transition line (between the free traffic and the coexisting phase) on the righthand side changes little for $a_{L}<1.0$. Also, the current does not change by $a_{T} / a_{L}$. Therefore, the current and stability

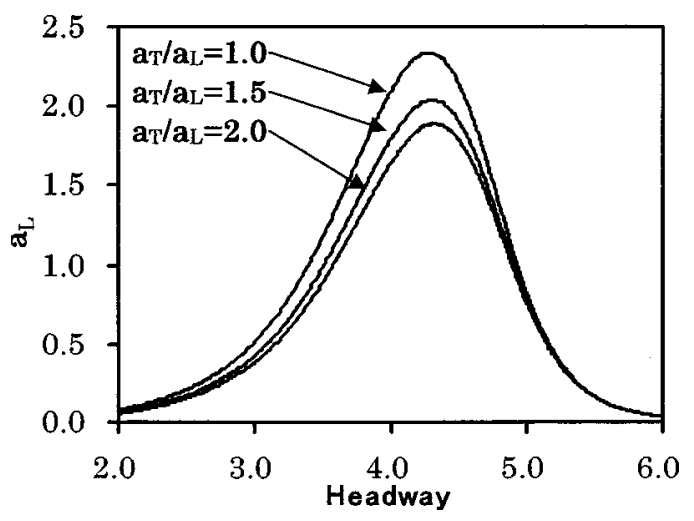

FIG. 4. Neutral stability curves for $a_{T} / a_{L}=1.0,1.5$, and 2.0 in the group movement of two vehicles per group where $v_{\max }=2.0$ and $x_{c}=3.0$. depend little on the sensitivity of automated vehicle and depend highly on the safety distance and the maximal velocity.

\section{SIMULATION}

We present the simulation result for the traffic flow with vehicles moving in groups. First, we consider the traffic flow for two vehicles per group. Second, we present the simulation result for five vehicles per group. Third, we consider the effect of a random configuration on the traffic behavior. We compare the traffic behavior of a random configuration with that of a periodic configuration.

\section{A. Two vehicles per group}

We carry out a computer simulation for vehicles moving in groups. We study the jamming transition points and the structure of traffic jams. The boundary is periodic. First, we study the group movement of two vehicles per group where the maximal velocity of automated vehicle is $v_{\max }=2.0$, the safety distance of automated vehicle is $x_{c}=3.0$, and the maximal velocity and safety distance of manual vehicle are twice those of automated vehicle, respectively. We calculate numerically Eq. (5) with optimal velocity functions (2) by using fourth-order Runge-Kutta method where the time interval is $\frac{1}{128}$. As a result, three types of traffic flow are distinguished similarly to the original optimal velocity model: (i) a free traffic with uniform velocity at low density, (ii) an inhomogeneous traffic at intermediate density in which jams appear, and (iii) a uniform-velocity congested phase. In the free traffic and congested traffic, the velocity profiles are uniform over all vehicles similarly to the original model. The headway changes alternately from manual vehicles to automated vehicles. The headway of manual vehicles is the same over all manual vehicles, the headway of automated vehicles is the same over all automated vehicles, and the headway of manual vehicles is different from that of automated vehicles. This point is different from that of the original optimal velocity model. Figure 5 shows (a) the velocity profiles and (b) the headway profiles of manual and automated vehicles for the free traffic where the sensitivity is $a=1.0$, the average headway is $\Delta x^{(0)}=5.5$, and the total number of vehicles is 100. We focus our attention on the inhomogeneous traffic. Figure 6 shows (a) the velocity profiles and (b) the headway profiles of manual and automated vehicles for the inhomogeneous traffic where the sensitivity is $a=1.0$ and the average headway is $\Delta x^{(0)}=4.25$. The density waves with kinkantikink form appear in the inhomogeneous traffic. The velocity profile of manual vehicles agrees with that of automated vehicles except for the left edge of the kink-antikink density waves. Each headway exhibits the kink-antikink density waves but the amplitude of manual vehicles is different from that of automated vehicles. Figure 7 shows the plot of velocity against headway after a sufficiently large time for each vehicle where the values of parameters are the same as in Fig. 6. The trajectories of all automated vehicles enter into a single orbit. All automated vehicles generate a single trajectory representing a limit cycle. Also, all manual vehicles generate a single trajectory different from that of automated 


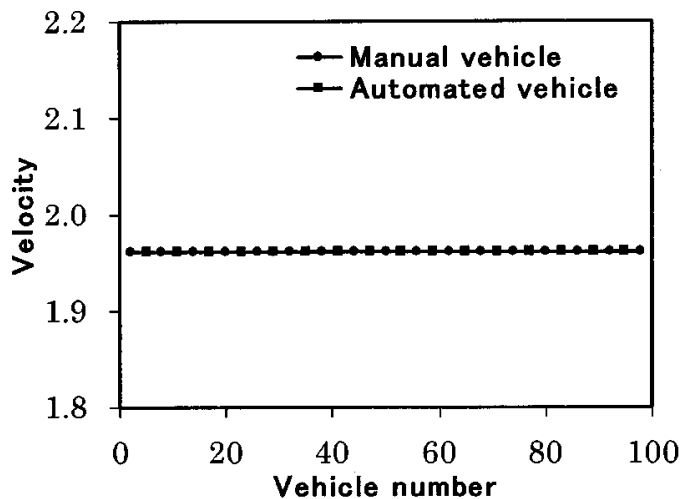

(a)

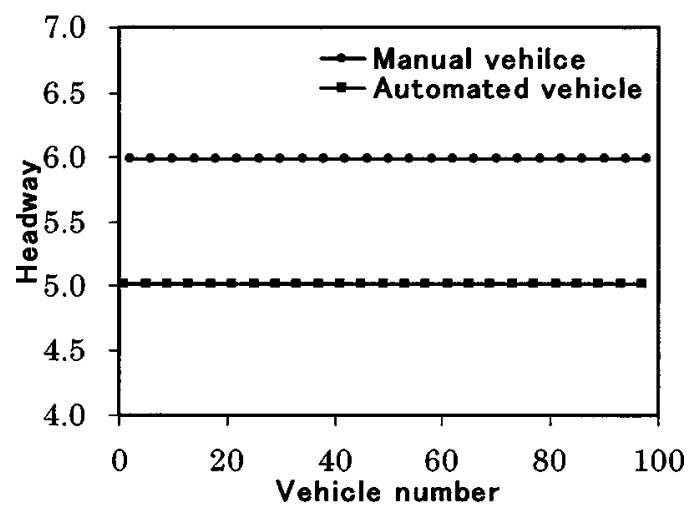

(b)

FIG. 5. (a) Velocity profiles of manual and automated vehicles and (b) headway profiles for the free traffic in the group movement of two vehicles per group, where the sensitivity is $a=1.0$, the average headway is $\Delta x^{(0)}=5.5, v_{\max }=2.0, x_{c}=3.0$, and the total number of vehicles is 100 .

vehicles. Each vehicle moves counterclockwise on the orbit. A small loop on the orbit of manual vehicles appears. This is due to generating spike of the velocity and headway profiles in Fig. 6.

We study the transition points among the free traffic, the inhomogeneous traffic, and the homogeneous congested traffic by varying sensitivity. Figure 8 shows the transition points obtained from simulation where $v_{\max }=2.0, x_{c}=3.0$, and the total number of vehicles is 100 . The simulation result is indicated by the square. The solid line represents the neutral stability curve of the case with two vehicles per group. The jamming transition line agrees with the neutral stability curve within numerical accuracy. The uniform-velocity traffic becomes unstable within the neutral stability curve and evolves to the inhomogeneous traffic.

We study the traffic current for the group movement of two vehicles per group. Figure 9 shows the plot of current against density where $a=1.0, v_{\max }=2.0, x_{c}=3.0$, and the total number of vehicles is 100 . The square indicates the simulation result. The solid line represents the currentdensity curve without jams. In the intermediate density region at which jams appear, the simulation result deviates from the solid curve. In the low-density region of the free traffic, the simulation result agrees with the current-density curve without jams. In the high-density region, the simula-

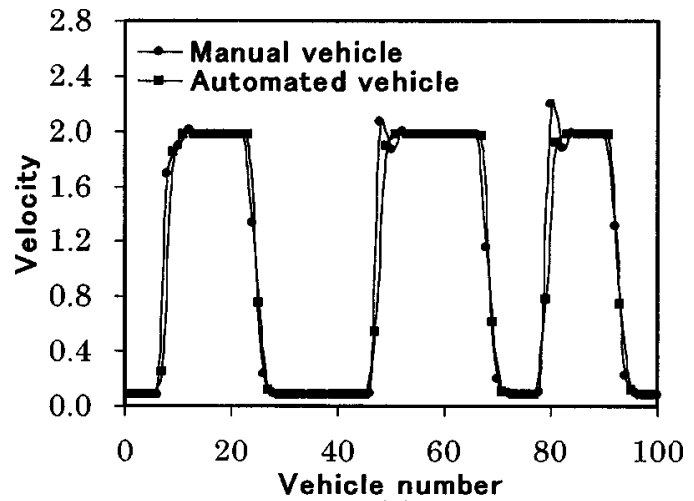

(a)

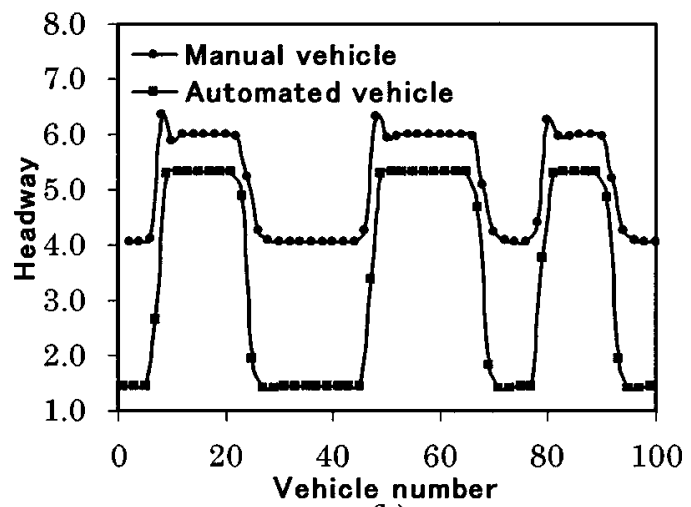

(b)

FIG. 6. (a) Velocity profiles of manual and automated vehicles and (b) headway profiles for the inhomogeneous traffic in the group movement of two vehicles per group where the sensitivity is $a$ $=1.0$, the average headway is $\Delta x^{(0)}=4.25, v_{\max }=2.0, x_{c}=3.0$, and the total number of vehicles is 100 .

tion result is consistent with the current-density curve without jams. Thus, the traffic current is enhanced at the intermediate and high-density regions by the group movement of two vehicles per group.

We study the effect of sensitivity of the automated vehicle on the jam structure. Figure 10 shows (a) the velocity pro-

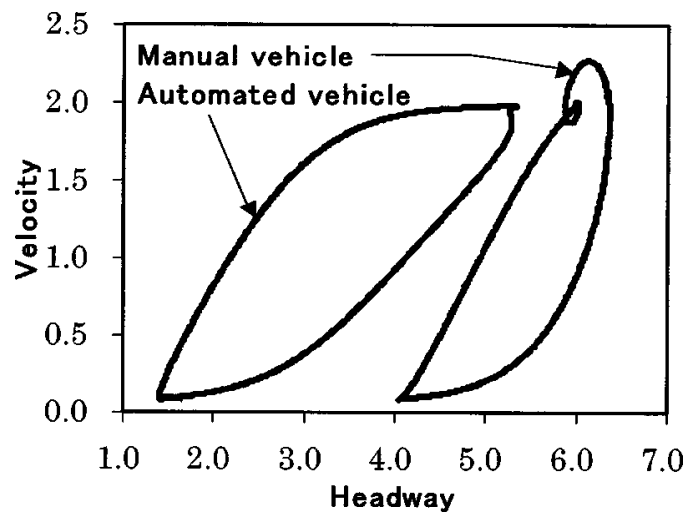

FIG. 7. Plot of velocity against headway after a sufficiently large time for each vehicle in the group movement of two vehicles per group, where the values of parameters are the same as Fig. 6. The loop on the left-hand side indicates the trajectory of automated vehicles. The loop on the right-hand side represents the trajectory of manual vehicles. 


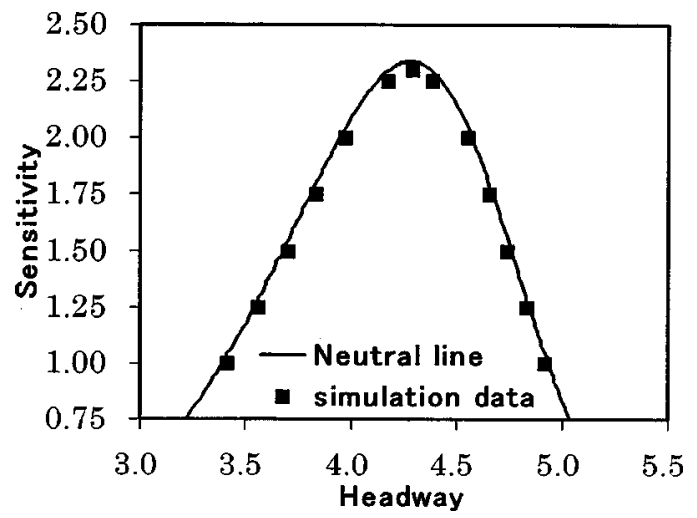

FIG. 8. Plot of transition points among the free traffic, inhomogeneous traffic, and homogeneous-congested traffic in the group movement of two vehicles per group in the headway-sensitivity space, where $v_{\max }=2.0$ and $x_{c}=3.0$. The square indicates the transition point obtained by simulation. The solid line represents the neutral stability curve.

files and (b) the headway profiles of manual and automated vehicles for the inhomogeneous traffic where the sensitivity of manual vehicle is $a_{L}=1.0$, the sensitivity of automated vehicle is $a_{T}=1.5$, and the average headway is $\Delta x^{(0)}$ $=4.25$. Figure 10 is compared with Fig. 6 of $a_{L}=a_{T}=a$ $=1.0$. The amplitudes of both headway and velocity out of and within the jam are nearly the same as those of Fig. 6.

Also, we show in Fig. 9 the plot of traffic current against density for $a_{L}=1.0$ and $a_{T}=1.5$. The triangular points indicate the simulation result. Those simulation points agree with the square points of $a_{L}=a_{T}=a=1.0$. Thus, the difference of the sensitivities does not affect the overall traffic behavior.

\section{B. Five vehicles per group}

We study the group movement of five vehicles per group where the maximal velocity of automated vehicle is $v_{\max }$ $=2.0$, the safety distance of automated vehicle is $x_{c}=3.0$,

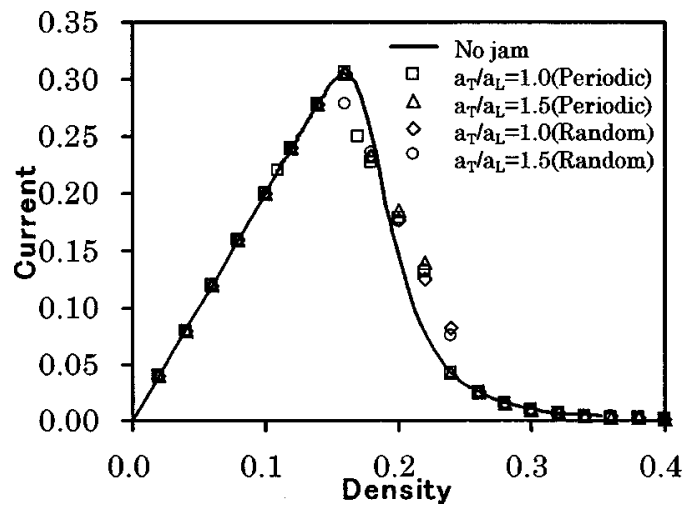

FIG. 9. Plots of current against density where $v_{\max }=2.0, x_{c}$ $=3.0$, and the total number of vehicles is 100. The square and triangle indicate, respectively, the simulation results for $a_{T} / a_{L}$ $=1.0$ and $a_{T} / a_{L}=1.5$ in the periodic configuration. The diamond and circle indicate, respectively, the simulation results for $a_{T} / a_{L}$ $=1.0$ and $a_{T} / a_{L}=1.5$ in the random configuration. The solid line represents the current-density curve without jams.

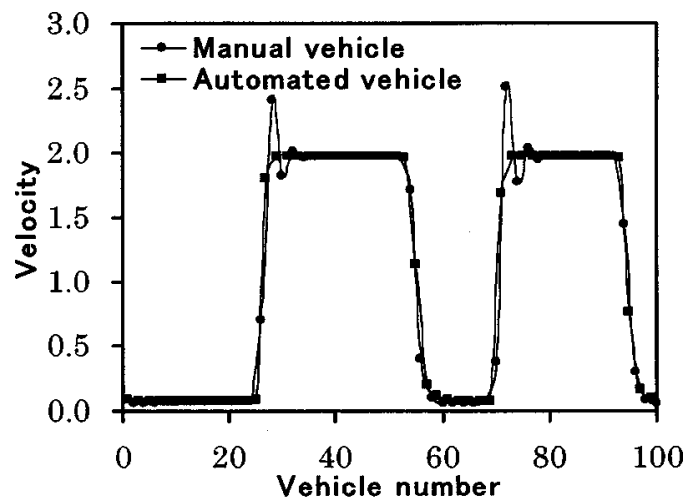

(a)

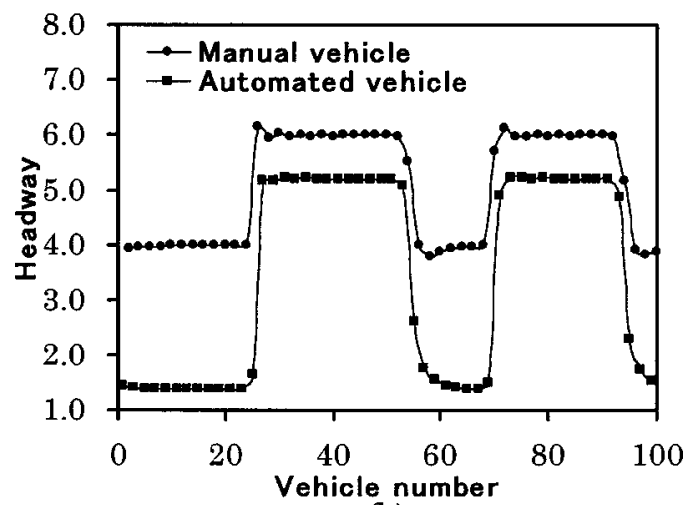

(b)

FIG. 10. (a) Velocity profiles of manual and automated vehicles and (b) headway profiles in the group movement of two vehicles per group for $a_{L}=1.0$ and $a_{T}=1.5$ where the average headway is $\Delta x^{(0)}=4.25, v_{\max }=2.0, x_{c}=3.0$, and the total number of vehicles is 100. Both profiles are compared with those in Fig. 6.

and the maximal velocity and safety distance of manual vehicle are twice those of automated vehicle, respectively. Similarly to the case of two vehicles per group, three types of traffic flow are distinguished: (i) a free traffic with uniform velocity at low density, (ii) an inhomogeneous traffic at intermediate density in which jams appear, and (iii) a uniform-velocity congested phase. In the free traffic and congested traffic, the velocity profiles are uniform over all vehicles similarly to the original model but the headway profiles are not homogeneous. The headway changes from manual vehicles to automated vehicles. The headway of manual vehicles is the same over all manual vehicles and the headway of automated vehicles is the same over all automated vehicles. We focus our attention on the inhomogeneous traffic. Figure 11 shows (a) the velocity profiles and (b) the headway profiles of manual and automated vehicles for the inhomogeneous traffic where the sensitivity is $a$ $=1.0$, the average headway is $\Delta x^{(0)}=3.5$, and the total number of vehicles is 200 . The leading manual vehicle within a group is labeled by 1 . The following automated vehicles are labeled in order by 2, 3, 4, and 5. The density waves with kink-antikink form appear in the inhomogeneous traffic. The velocity profile of manual vehicles agrees with that of automated vehicles except for the left edge of the kink density waves. Each headway exhibits the kink-antikink density 


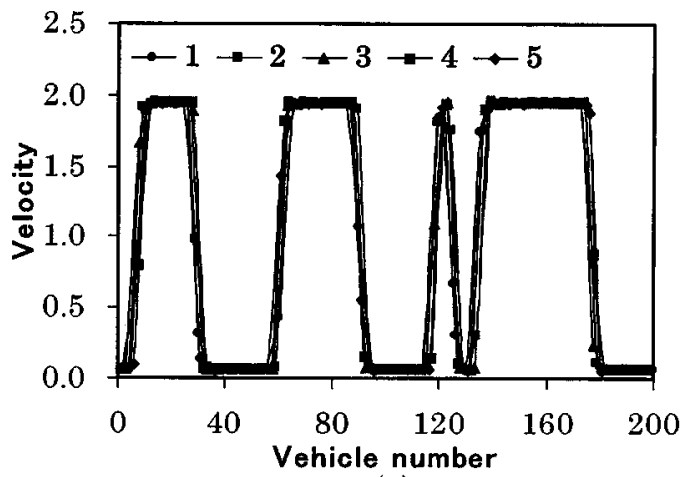

(a)

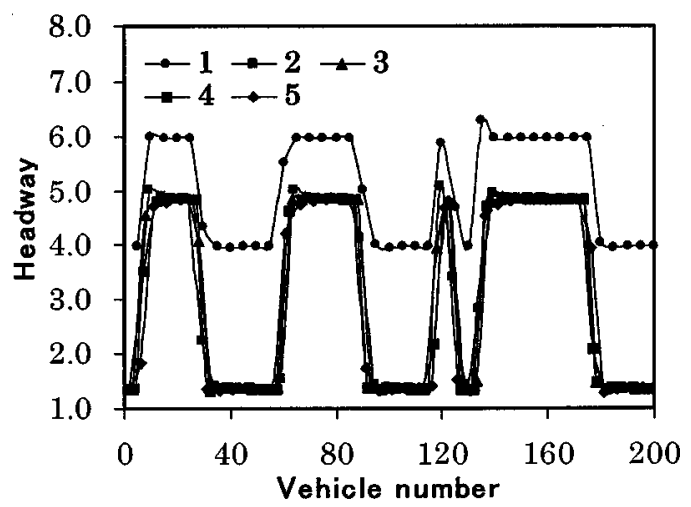

(b)

FIG. 11. (a) Velocity profiles of manual and automated vehicles and (b) headway profiles for the inhomogeneous traffic in the group movement of five vehicles per group, where the sensitivity is $a$ $=1.0$, the average headway is $\Delta x^{(0)}=3.5, v_{\max }=2.0, x_{c}=3.0$, and the total number of vehicles is 200 .

waves but the amplitude of manual vehicles is different from that of automated vehicles. Figure 12 shows the plot of velocity against headway after a sufficiently large time for five vehicles where the values of parameters are the same as Fig. 11. Each trajectory of five vehicles enters into a single orbit and their trajectories are different between each other. Each

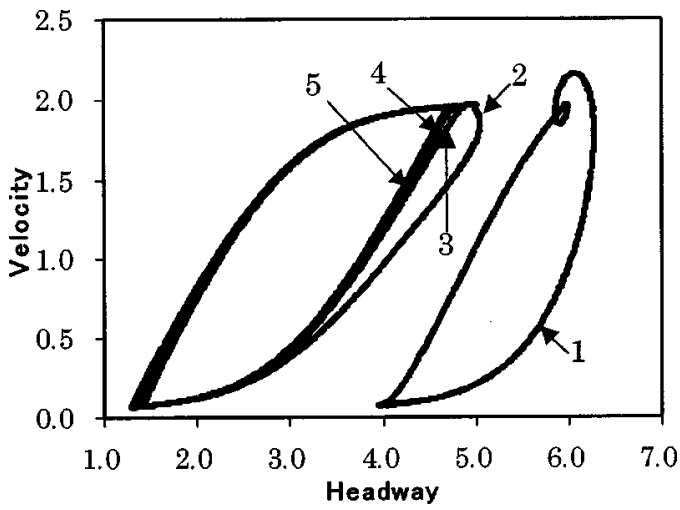

FIG. 12. Plot of velocity against headway after a sufficiently large time for each vehicle in the group movement of five vehicles per group, where the values of parameters are the same as those in Fig. 11. The vehicles within a group are numbered $1-5$ from the top to the back.

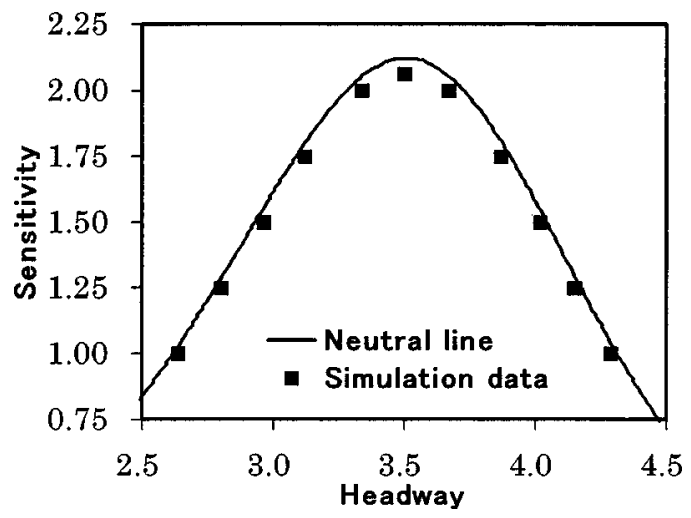

FIG. 13. Plot of transition points among the free traffic, inhomogeneous traffic, and homogeneous-congested traffic in the group movement of five vehicles per group in the headway-sensitivity space, where $v_{\max }=2.0$ and $x_{c}=3.0$. The square indicates the transition point obtained by simulation. The solid line represents the neutral stability curve.

vehicle generates a single trajectory representing a limit cycle. Each vehicle moves counterclockwise on the orbit. A small loop on the orbit of manual vehicles appears. This is due to generating spike of the velocity and headway profiles in Fig. 11. The trajectory of the automated vehicle just behind the manual vehicle is closer to the trajectory of the manual vehicle. The trajectories of the other automated vehicles are in agreement with each other.

In the case of five vehicles per group, we study the transition points among the free traffic, the inhomogeneous traffic, and the homogeneous congested traffic by varying sensitivity. Figure 13 shows the transition points obtained from simulation where $v_{\max }=2.0, x_{c}=3.0$, and the total number of vehicles is 200 . The simulation result is indicated by the square. The solid line represents the neutral stability curve of five vehicles. The jamming transition line agrees with the neutral stability curve within numerical accuracy. The uniform-velocity traffic becomes unstable within the neutral stability curve and evolves to the inhomogeneous traffic.

We study the traffic current for the group movement of five vehicles. Figure 14 shows the plot of current against

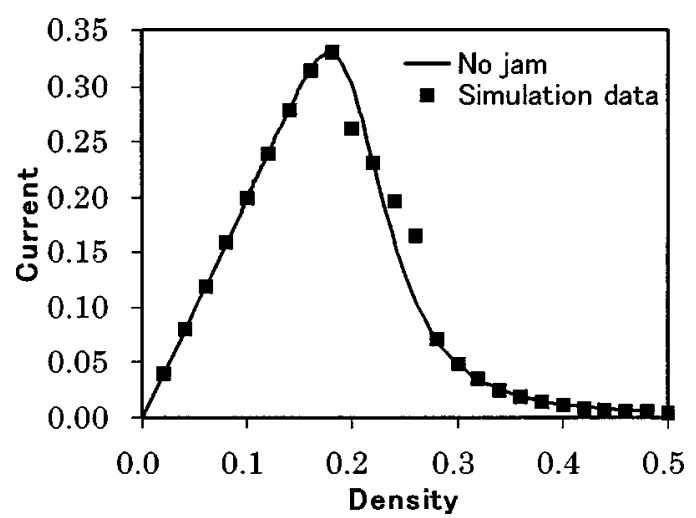

FIG. 14. Plot of current against density where $a=1.0, v_{\max }$ $=2.0, x_{c}=3.0$, and the total number of vehicles is 100 . The square indicates the simulation results. The solid line represents the current-density curve without jams. 
density where $a=1.0, v_{\max }=2.0, x_{c}=3.0$, and the total number of vehicles is 200 . The square indicates the simulation result. The solid line represents the current-density curve without jams. In the intermediate density region at which jams appear, the simulation result deviates from the solid curve. In the low-density region of the free traffic, the simulation result agrees with the current-density curve without jams. In the high-density region, the simulation result is consistent with the current-density curve without jams. Similarly to the case of two vehicles per group, the traffic current is enhanced at the intermediate and high-density regions by the group movement. Thus, we find that the enhancement effect of current and the stabilization effect increase with the number per group.

\section{Random configuration}

Finally, we consider the binary mixture of vehicles with a random configuration. We study the traffic behavior numerically. We choose manual vehicles randomly with probability $\frac{1}{2}$ from all vehicles. We assign the automated vehicles to the remaining vehicles. The maximal velocity of automated vehicle is $v_{\max }=2.0$, the safety distance of automated vehicle is $x_{c}=3.0$, and the maximal velocity and safety distance of manual vehicle are twice those of automated vehicle. Similarly to the case of periodic configuration, three types of traffic flow are distinguished: (i) a free traffic with uniform velocity at low density, (ii) an inhomogeneous traffic at intermediate density in which jams appear, and (iii) a uniformvelocity congested traffic. In the free traffic and congested traffic, the velocity profiles are uniform over all vehicles similarly to the periodic case. The headway changes from manual vehicles to automated vehicles. The headway of manual vehicles is the same over all manual vehicles and the headway of automated vehicles is the same over all automated vehicles. We focus our attention on the inhomogeneous traffic. Figure 15 shows (a) the velocity profiles and (b) the headway profiles of manual and automated vehicles for the inhomogeneous traffic where the sensitivity is $a$ $=1.0$, the average headway is $\Delta x^{(0)}=4.25$ and the total number of vehicles is 100 . The density waves with irregular kink form appear in the inhomogeneous traffic. The velocity profile of manual vehicles agrees roughly with that of automated vehicles except for the left edge of the kink density waves. Similarly to the periodic case, each headway exhibits the kink-antikink density waves but the amplitude of manual vehicles is very different from that of automated vehicles. Among automated vehicles, each headway profile is a little different from the other. Also, each headway profile of manual vehicles is a little different from the other. The difference among automated vehicles depends on the configuration of vehicles.

We study the traffic current for the random configuration in which the number of manual vehicles equals that of automated vehicles. Figure 9 shows the plot of current against density where $a=1.0, v_{\max }=2.0, x_{c}=3.0$, and the total number of vehicles is 100 . The diamond point indicates the simulation result for $a_{L}=a_{T}=a=1.0$. The circle indicates the simulation result for $a_{L}=1.0$ and $a_{T}=1.5$. The solid line

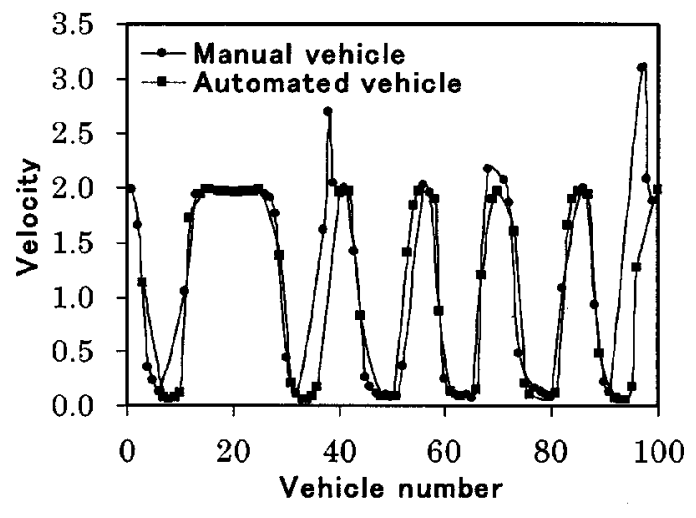

(a)

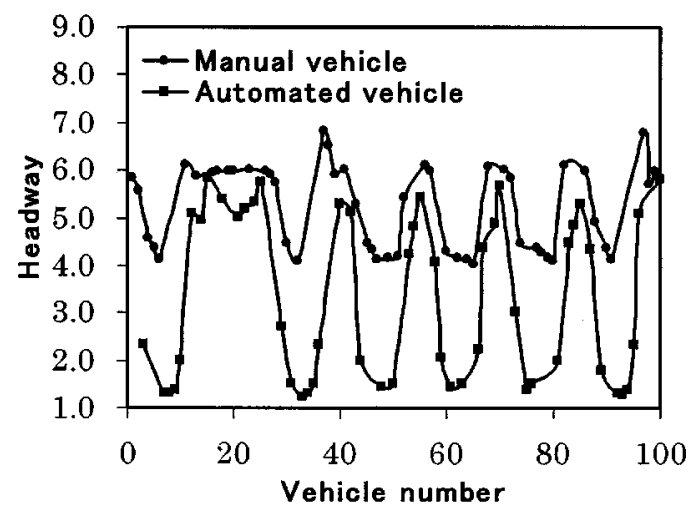

(b)

FIG. 15. (a) Velocity profiles and (b) headway profiles of manual and automated vehicles for the inhomogeneous traffic in the binary mixture of vehicles, where the sensitivity is $a=1.0$, the average headway is $\Delta x^{(0)}=4.25, v_{\max }=2.0, x_{c}=3.0$, and the total number of vehicles is 100 .

represents the current-density curve of two vehicles without jams. The simulation result agrees with the current-density curve without jams except for the inhomogeneous traffic region at an intermediate density. The current with the random configuration is consistent with that of the periodic case with two vehicles per group, except for the neighborhood of the transition points. The transition points depend weakly on the random configuration. The enhancement of current by the group movement changes little in both cases of the random and periodic configurations. Therefore, we find that the group movement has a significant effect on the enhancement of traffic current irrespective of the configuration.

In a different model, two vehicle types, there called cars and trucks, have been studied in a random configuration by Treiber, Hennecke, and Helbing [33]. They have shown that the trucks have a significant effect on the traffic flow.

\section{SUMMARY}

We have investigated the traffic behavior of group movement by extending the car-following model. We have shown that the group movement stabilizes the traffic flow and the traffic current is enhanced significantly without jams. We have studied the effect of the group movement on the jam- 
ming transition. We have shown the structure of the jams in the group movement. We have analyzed the traffic behavior by the use of the linear stability method. We have derived analytically the neutral stability condition. We have compared the transition line among the homogeneous free traffic, the inhomogeneous traffic (coexisting phase), and the homogeneous congested traffic with the neutral stability curve. We have found that the transition line is consistent with the neutral stability curve. We have investigated the traffic behavior in the binary mixture of vehicles with a random configuration. We have shown that the current-density curve of the random case agrees strongly with that of the periodic case.

It is expected that our model and result will serve the intelligent transportation system in the near future.
[1] D. Chowdhury, L. Santen, and A. Schadschneider, Phys. Rep. 329, 199 (2000).

[2] D. Helbing, Verkehrsdynamik (Springer, Berlin, 1997).

[3] Traffic and Granular Flow 97, edited by M. Schreckenberg and D. E. Wolf (Springer, Singapore, 1998).

[4] S. Krauss, P. Wagner, and C. Gawron, Phys. Rev. E 55, 5597 (1997).

[5] T. Nagatani, Phys. Rev. E 58, 4271 (1998).

[6] T. Nagatani, Phys. Rev. E 59, 4857 (1999).

[7] D. Helbing, A. Hennecke, and M. Treiber, Phys. Rev. Lett. 82, 4360 (1999).

[8] H. Y. Lee, H. W. Lee, and D. Kim, Phys. Rev. E 59, 5101 (1999).

[9] M. Treiber, A. Hennecke, and D. Helbing, Phys. Rev. E 62, 1805 (2000).

[10] T. Nagatani, Physica A 280, 602 (2000).

[11] G. B. Whitham, Proc. R. Soc. London, Ser. A 428, 49 (1990).

[12] K. Nagel and M. Schreckenberg, J. Phys. I 2, 2221 (1992).

[13] M. Bando, K. Hasebe, A. Nakayama, A. Shibata, and Y. Sugiyama, Phys. Rev. E 51, 1035 (1995).

[14] B. S. Kerner, P. Konhauser, and M. Schilke, Phys. Rev. E 51, 6243 (1995).

[15] B. S. Kerner and H. Rehborn, Phys. Rev. E 53, R1297 (1996).

[16] O. Biham, A. A. Middleton, and D. A. Levine, Phys. Rev. A 46, R6124 (1992).

[17] T. Nagatani, Phys. Rev. E 48, 3290 (1993).
[18] J. A. Cuesta, F. C. Matinez, J. M. Nolera, and A. Sanchez, Phys. Rev. E 48, 4175 (1993).

[19] K. Nagel, D. E. Wolf, P. Wagner, and P. Simon, Phys. Rev. E 58, 1425 (1998).

[20] D. Helbing and M. Schreckenberg, Phys. Rev. E 59, R2505 (1999).

[21] D. Helbing, Phys. Rev. E 53, 2366 (1996).

[22] M. Treiber, A. Hennecke, and D. Helbing, Phys. Rev. E 59, 239 (1999).

[23] K. Nagel, Phys. Rev. E 53, 4655 (1996).

[24] T. Nagatani, Phys. Rev. E 61, 3534 (2000).

[25] T. Nagatani, Phys. Rev. E 60, 6395 (1999).

[26] B. S. Kerner, P. Konhauser, and M. Schilke, Phys. Lett. A 215, 45 (1996).

[27] D. Helbing and M. Treiber, Phys. Rev. Lett. 81, 3042 (1998).

[28] T. Komatsu and S. Sasa, Phys. Rev. E 52, 5574 (1995).

[29] M. Muramatsu and T. Nagatani, Phys. Rev. E 60, 180 (1999).

[30] T. Fujioka, M. Omae, and K. Miyake, Proceedings of the 6th Symposium on Simulation of Traffic Flow, 1999 (unpublished), p. 24.

[31] A. Bose, Proceedings of the 1999 American Control Conference, 1999 (unpublished), p. 233.

[32] T. Kawabe, IEEE ITSC 1997, 397 (1997).

[33] M. Treiber, A. Hennecke, and D. Helbing, in Traffic and Granular Flow '99, edited by D. Helbing, H. J. Herrmann, M. Schreckenberg, and D. E. Wolf (Springer, Berlin, 2000), p. 365. 